\title{
Diseño y evaluación de un instrumento para medir el uso de internet en la era COVID-19 *
}

\author{
Design and Evaluation of an Instrument to Measure Internet use During the \\ COVID-19 Era
}

\author{
Cruz García-Lirios \\ Posdoctorante en Ciencias de la Complejidad, Universidad Autónoma del Estado de México, \\ Toluca-México, cgarciali@uaemex.mx
}

José Marcos Bustos-Aguayo

Doctor en Psicología, Universidad Nacional Autónoma de México, Ciudad de México-México, marcos.bustos@unam.mx

\begin{abstract}
Cómo citar / How to cite
García-Lirios, C.; Bustos-Aguayo, J. M. (2021). Diseño y evaluación de un instrumento para medir el uso de internet en la era COVID-19. Revista CEA, v. 7, n. 14, e1665. https://doi.org/10.22430/24223182.1665
\end{abstract}

Recibido: 10 de diciembre de 2020

Aceptado: 17 de abril de 2021

\section{Resumen}

En el marco de la pandemia ocasionada por el nuevo coronavirus SARS-CoV-2 y la enfermedad COVID19, las políticas de contención y mitigación de la pandemia consisten en el confinamiento y el distanciamiento social. En esa estrategia, el uso de Internet ha sido fundamental para el trabajo a distancia, aunque la medición de la actividad internauta apenas está en etapa de estudio. El objetivo del presente trabajo fue el diseño y validación de un instrumento para medir el uso de Internet en la era COVID-19. Para ello se aplicó una encuesta a 253 estudiantes de la Universidad Autónoma del Estado de México. Inicialmente, para verificar la validez de contenido de los ítems considerados en la encuesta, se realizó un análisis Delphi, y para verificar la validez de los constructos, se procedió con el cálculo de estadísticos como Alfa de Cronbach, KMO, ji-cuadrado, entre otros. Finalmente, se dio paso con un análisis factorial confirmatorio para corroborar las ocho dimensiones identificadas previamente en la literatura: selectividad, compatibilidad, computabilidad, accesibilidad, extensionalidad, acumulatividad, ansiedad y adicción, las cuales logran explicar el 59\% de la varianza total expuesta. Aunque estos hallazgos solo se aplican a la muestra, los resultados obtenidos en el presente trabajo proporcionan evidencias acerca de las propiedades psicométricas del instrumento y sigue abriendo paso para el contraste en otros escenarios y con otras muestras de estudio.

Palabras clave: análisis factorial confirmatorio, método Delphi, validez del constructo.

\footnotetext{
* Este artículo se deriva del proyecto titulado «Gobernanza de las Tecnologías de Información y Comunicación» y ha sido financiado con recursos de la Red Académica Multidisciplinar, número de registro: ATN-CGL-2020-0001.
} 
Clasificación JEL: L86.

\title{
Highlights
}

- La pandemia y el uso de Internet coexisten en un escenario de utilidad y fácil acceso al uso de tecnologías, dispositivos y redes electrónicas.

- Los determinantes del uso de Internet han sido identificados como variables cognitivas que regulan o moderan el impacto de la información diseminada en redes digitales.

- El uso de Internet es el resultado del impacto y la información circundante en las redes digitales, así como de la filtración que supone su utilidad y fácil accesibilidad.

\begin{abstract}
In the context of the pandemic caused by the new SARS-CoV-2 coronavirus and the COVID-19 disease, the policies to contain and mitigate it consist of isolation and social distancing. As part of these strategies, internet use has been fundamental for telecommuting; however, measuring internet activity is an incipient field of research. The aim of this study is to design and evaluate an instrument to measure internet use during the COVID-19 era. For that purpose, a survey was conducted with 253 students from the Universidad Autónoma del Estado de México. To verify the validity of the content of the items included in the survey, a Delphi analysis was carried out; and, to verify the validity of the constructs, several statistics were calculated, e.g., Cronbach's alpha, KMO, and chi-squared, among others. Finally, a confirmatory factor analysis confirmed the eight dimensions that had been previously identified in the literature: selectivity, compatibility, computability, accessibility, extensionality, cumulativeness, anxiety, and addiction. They explain $59 \%$ of the total variance that was found. Although these findings are applicable only to this sample, the results obtained in this study provide evidence of the psychometric properties of the instrument, which can be used to make comparisons with other contexts and samples.
\end{abstract}

Keywords: Confirmatory factor analysis, Delphi method, construct validity.

JEL classification: L86.

\section{Highlights}

- The pandemic and internet use coexist in a scenario of usefulness and easy access to technologies, devices, and electronic networks.

- Internet use determinants have been identified as cognitive variables that regulate or moderate the impact of the information disseminated in digital networks.

- Internet use is the result of the impact of and the information circulating in digital networks, as well as the filtering that makes them useful and easily accessible.

\section{INTRODUCCIÓN}

Al momento de escribir el presente trabajo, la pandemia del SARS-CoV-2 y la enfermedad COVID-19 habían dejado dos millones de víctimas mortales en el mundo (Organización Mundial de la Salud, 2021). Los ministros de salud, como parte de un control de calidad en el conteo y la autocorrección 
de síntomas, han reconocido decesos catalogados como neumonías atípicas (Cayla, 2009). En ese proceso de gestión, control y manejo de la crisis sanitaria a través de boletines, conferencias, entrevistas o comunicados, han puesto de relieve el sentido político y propagandístico de la cuestión, soslayando los avances científicos y tecnológicos.

Se trata de la difusión de estrategias y programas de gobierno que delegan en el individuo la responsabilidad del contagio al inducir su confinamiento y aislamiento sin considerar sus ocupaciones o estilos de vida, o bien, sus efectos en niveles de estrés, ansiedad y depresión (Ozamis et al., 2020). Mientras que la ciencia avanza en la decodificación de la transmisión e impacto del virus en el sistema inmunológico, los gobiernos apostaron por estrategias de confinamiento y distanciamiento social hasta la aprobación de las vacunas, reduciendo significativamente los contagios, enfermedades y muertes.

De este modo, la comunicación de la crisis sanitaria, diseminada en los medios tradicionales y electrónicos, ha propiciado la polarización de las audiencias al generar malestar por la difusión de infectados, enfermos y muertes frente a la postura defensiva de las autoridades quienes han desviado en las críticas su planteamiento del problema: hay más casos porque los medios desinforman, confundiendo a sus audiencias sobre las estrategias del Estado ante la crisis sanitaria y económica.

En este contexto, Internet ha sido un medio y un emisor de datos que confrontan o confirman la propaganda del Estado. En el marco de la pandemia, la información antecede a las decisiones de consumo, uso y apropiación de espacios. Por consiguiente, el estudio del uso de Internet ha tenido un interés cada vez más creciente en la ciencia de los datos. Esto es así porque el análisis del uso de la web devela las diferencias entre procesos tangibles e intangibles, como es el caso de las asimetrías entre clientes de bancos y usuarios de la banca electrónica (Yasa et al., 2014).

El objetivo del presente trabajo fue el diseño y validación de un instrumento para medir el uso de Internet en la era COVID-19, considerando dimensiones alusivas a la intención de encontrar la información teniendo en cuenta el proceso de selección e intercambio de contenidos académicos disponibles en línea. Para ello se revisó la literatura y, con base en la escala de estado de conocimiento, se construyó la búsqueda de información de intención con fines académicos.

La premisa que guía esta investigación alude a que el uso de Internet puede ser observado en diferentes factores relativos a su efecto en la agenda pública, como es el caso de la pandemia y las políticas de mitigación y contención (Liao et al., 2018). En este sentido, las dimensiones del uso de Internet ayudarían a evitar la propagación de la pandemia a partir del establecimiento de una agenda volátil en cuanto a promoción de la salud y prevención de riesgos a través del distanciamiento y confinamiento social. Incluso, ayudaría a la mitigación y contención de la crisis sanitaria a partir de dimensiones que anteceden a contagios, enfermedades y muertes como es el autocuidado difundido en los medios de comunicación. 


\section{MARCO TEÓRICO}

\section{Panorama en torno al uso de Internet en el mundo}

El uso de tecnologías, dispositivos y redes electrónicas no sólo genera diferencias entre usuarios, sino también incluso entre aquellos que utilizan tecnologías que con el tiempo perfeccionan y que implica el aprendizaje continuo y el procesamiento del conocimiento y la difusión de la información (Drozdova, 2020; Cervantes-López et al., 2020). Como parte de la sociedad de la información, el uso intensivo de tecnología y dispositivos electrónicos ha exacerbado las diferencias entre las redes digitales y los usuarios que tienen acceso (Kleine, 2009; Mayor-Ríos et al., 2019). La brecha digital entre generaciones e incluso entre usuarios hace necesario conciliar intereses, necesidades $y$ expectativas, así como habilidades y conocimientos de acuerdo con las oportunidades y capacidades, demandas y recursos locales disponibles para los requerimientos del mercado internacional (Wamuyu, 2017; Zhang et al., 2020). El sistema en el que se resuelven estas diferencias es la gobernanza, y el instrumento por el que se ponderan las asimetrías y similitudes se conoce como agenda pública, aunque en el ámbito tecnológico se conoce como ecosistema digital. Se trata de exclusión e inclusión de usuarios a partir de las contradicciones con respecto a un sistema digital (Gard, 2018). Es el caso de quienes no adoptan los dispositivos digitales y prefieren los analógicos.

Por lo tanto, si un país tiene una agenda enfocada en redes, tecnologías y electrónica como la República de Estonia, es de esperar una pequeña brecha entre generaciones, pero muy contraria a casos como el de la República de Etiopía. Si la agenda digital es casi inexistente, la disponibilidad, el acceso y la conectividad son casi nulos, entonces es posible esperar una brecha digital entre sus generaciones e incluso entre los pocos usuarios de Internet, aún y cuando su uso se ha diseminado en mayor medida por el marketing dirigido al cliente minorista (Sevim et al., 2017).

Las expectativas de crecimiento económico, tecnológico y comercial permiten predecir la estrecha relación entre la tendencia de crecimiento poblacional y el consumo electrónico. En la medida que aumente la población, las expectativas del uso de las tecnologías de la información y la comunicación estarán más orientadas a la compra y venta de productos y la contratación de servicios en Internet (Gravili et al., 2018). Para estos países, se espera un crecimiento del uso de tecnologías electrónicas. Sin embargo, el trabajo de la población supone consumir Internet en mayor medida. En cuanto a la edad productiva, India seguirá con la mayor fuerza laboral con cerca de 998 millones; México, en el sexto lugar, con 73 millones; e Islandia ocupará el último lugar con alrededor de 215000 trabajadores. Sin embargo, una consecuencia de la dinámica del crecimiento poblacional es la vejez que, en el caso de los países mencionados, tendrá la misma tendencia. En India habrá alrededor de 222 millones de ancianos, en México 29 millones e Islandia tendrá 108000 personas mayores de 65 años (Mecinas Montiel, 2016). Si el pronóstico sobre la tendencia de crecimiento poblacional, oferta educativa y acceso a internet para México se mantiene constante hasta 2050, las variables predicen indirectamente el uso de una tecnología a través de intenciones, actitudes y percepciones. Este sentido multidisciplinar permite la observación sistemática de Internet (Garduño Vera, 2004). En torno a la eficiencia del uso de Internet, el sexo y la edad se consideran factores externos que inciden a través de percepciones, actitudes e intenciones indirectas sobre el consumo electrónico (Arribas \& Islas, 2009). Los estudios psicológicos de Internet tienen modelos desarrollados para predecir la eficiencia estructural en torno a su uso desde variables sociodemográficas. 


\section{Panorama en torno al uso de Internet en México}

La tendencia general de la población al uso de Internet se puede explicar de forma prospectiva a partir de la tendencia actual y la base local. En el caso de México, las localidades con más de 2500 habitantes muestran un aumento significativo en relación con las localidades con más de 15000 habitantes (Instituto Nacional de Estadística, Geografía e Informática, 2020). Mientras que en las ciudades pequeñas aumenta la tasa de crecimiento, en las grandes tiende a disminuir. Estos datos, combinados con la tendencia educativa, explican el uso de Internet con fines comerciales más que educativos o laborales. En México la cobertura disminuye, pero la oferta aumenta en instituciones privadas, principalmente en ciudades con más de un millón de habitantes, ampliando el cubrimiento educativo en sus localidades, donde el nivel más alto registró un crecimiento espurio. Sin embargo, el acceso a Internet tiene una importancia significativa en las ciudades medianas y pequeñas. Esto es así porque los usuarios, aunque tienen menos experiencia en el uso de Internet, conocen otras ciudades a través de Internet y las redes electrónicas, generando mayores experiencias positivas hasta alcanzar la satisfacción, la cual predice la facilidad y la utilidad percibida de la tecnología (Colesca \& Dobrica, 2008).

Las tasas de esperanza de vida, educación, alfabetización, producto interno bruto (PIB), matrícula educativa y desarrollo humano son parámetros de crecimiento, siendo Ciudad de México la entidad con mayor esperanza de vida hasta los 77 años, seguida de Nuevo León (Peláez Herreros, 2009). En contraste, Chiapas y Oaxaca están en el último lugar con 72 años. Se pueden observar cinco años de diferencia entre las entidades. Existe una relación entre el nivel de esperanza de vida, el analfabetismo y la escolaridad en cada uno de los 32 estados si se consideran los porcentajes de analfabetismo y escolaridad entre las entidades. Solo en el caso de Chiapas, Guerrero y Oaxaca los porcentajes son inferiores al 80 por ciento para los casos de personas mayores de 15 años. Sin embargo, esas diferencias en los porcentajes de escolaridad varían tres puntos porcentuales. En cambio, si se considera el PIB per cápita, el Distrito Federal alcanza los 18000 dólares estadounidenses. Los datos utilizados respaldan los niveles de desarrollo humano, donde Chiapas, Guerrero y Oaxaca nuevamente comparten el último lugar, con una diferencia de 18 puntos porcentuales con respecto al Distrito Federal. Un aumento en cada variable, corresponde a un crecimiento en las demás, incluso en el caso de analfabetismo, que debería disminuir con el incremento de la escolaridad, el PIB o el desarrollo humano. En este sentido, el consumo también podría ser una variable correlacionada con los índices expuestos. En el caso específico de consumo de la electrónica, México se encuentra dentro de los países con la banda ancha y el acceso a Internet más limitados. En el caso del Distrito Federal, sus niveles de crecimiento podrían estar relacionados con los de consumo electrónico.

Estos datos demográficos muestran un rasgo de la Población Económica Activa (PEA) sin tener en cuenta su relación con la tecnología y la Internet. En cada uno de los sectores, educativo o empresarial, la tecnología aumenta la productividad de la organización, y con ello aumenta el desempeño laboral de los trabajadores. Sin embargo, las Tecnologías de la Información y la Comunicación (TIC), principalmente Internet, están relacionadas con el sector terciario. En este sentido, los estudios psicológicos de la aceptación de las TIC han establecido variables e intenciones determinantes del uso de Internet. Los datos presentados muestran tendencias de crecimiento en población, educación e Internet. Estas cifras apoyan la hipótesis sobre la formación en tecnología de la información y la comunicación, sobre todo del servicio de internet, específicamente el comercio 
electrónico. Sin embargo, debido a que la mayoría de los usuarios son menores de 19 años, el consumo electrónico parece estar enfocado exclusivamente para redes sociales (Eddine Toudert, 2015,). En este sentido, los estudios psicológicos han utilizado dos modelos teóricos para demostrar la hipótesis de la actitud hacia el uso de Internet.

\section{Teoría del uso de Internet}

El estado del conocimiento ha posicionado y desarrollado tres modelos para explicar el impacto de la tecnología socioeconómica sobre las variables cognitivas y de comportamiento organizacional. Son modelos predominantemente cognitivos los que explican y predicen las decisiones y acciones de aceptación o rechazo de las TIC, aunque coexisten con aproximaciones normativas y valorativas (Jiang et al., 2010).

El primer modelo explica los procesos psicológicos deliberados que llevan a los individuos a ejecutar una acción razonada. La Teoría de la Acción Razonada (TRA, por sus siglas en inglés) incluye ocho variables que predicen causalmente una acción específica y premeditada (Lai, 2017). Las variables sociotecnológicas impactan creencias, valoraciones y motivaciones en la medida en que aumentan su grado de fomento a la innovación y expectativas de los resultados de su uso sistemático. En este sentido, los usuarios forman creencias sobre los usos de la tecnología y el impacto en su estilo de vida diaria. La tecnología, con una valoración positiva, aumenta las expectativas de durabilidad y las motivaciones de uso entre los usuarios. La interrelación entre estos factores repercute en sus disposiciones para quienes utilizan la tecnología y los principios que rigen su empleo. Ambas variables trasladarán sus efectos en las decisiones sobre el uso de la tecnología. Este es un punto especial del proceso deliberado ya que las decisiones incrementarán su uso sistemático.

Precisamente, en cuanto a la sistematización de la conducta deliberada, la TRA incluye variables exógenas a creencias y regulaciones que dan lugar al control del modelo. A diferencia de esta, la Teoría del Comportamiento Planificado (TPB, por sus siglas en inglés) solo considera a las creencias como transmisoras de efectos sociotecnológicos sobre la cognición y el comportamiento humano (Primabudi \& Samopa, 2017). Esta diferencia es importante porque la TRA solo explica el sistema racional y los individuos premeditados en un nivel generalizado de abstracción en el que se asume que existen relaciones causales entre los mitos y costumbres sobre las conductas. Por el contrario, la TPB plantea la predicción de una acción específica de sistematización, creencias y expectativas en situaciones limitadas. Dado que la racionalidad deliberada es un requisito de la planificación, la TRA es un antecedente de la TPB. Teniendo en cuenta el uso sistemático de las TIC, tendría entonces que anteponer su grado de deliberación y, especialmente, de planificación. Estos dos modelos explican los procesos racionales y las sistemáticas creencias predictoras de comportamientos delimitados, percepciones, actitudes e intenciones. La TRA y la TPB excluyen las variables demográficas indirectas como determinantes del comportamiento. Sin embargo, la TPB incluye percepciones de control variables para remitir la opinión de las personas sobre la facilidad o dificultad de realizar una acción planificada en un área designada. El control de la conducta percibida está relacionado con la variable eficiencia de percepción y la variable percepción de facilidad de uso de la tecnología.

La TPB también incluye la percepción de control del comportamiento a predecir. En situaciones específicas, los individuos tienden a repetir sus acciones e internalizarlas como parte de su vida cotidiana. La TPB intenta explicar este proceso de internalización proponiendo expectativas de 
control de la conducta (Zahid \& Haji Din, 2019). En la medida en que los comportamientos puedan ser repetibles y sus resultados favorables, los individuos llevarán a cabo una acción si está dentro de sus posibilidades de control y de sus expectativas de logro. La TPB incluye las relaciones causales y lineales entre las variables. Se pueden observar rutas de relación que parten de creencias y terminan en comportamiento. En este proceso, la actitud hacia el comportamiento, las variables de control estándar y la percepción subjetiva es la mediación de los efectos de las creencias relativas a las normas de comportamiento y control. Solo en el caso de expectativas de control real, la relación es directa con respecto al comportamiento. En el caso del uso de las TIC, si los usuarios tienen creencias positivas sobre el uso de la tecnología, entonces sus evaluaciones, expectativas y principios determinarán sus decisiones de consumo de tecnología.

La Teoría de la Autoeficacia (TSE, por sus siglas en inglés) incluye la autoevaluación de las capacidades operativas que determinan el comportamiento de las personas, sus patrones de pensamiento y emociones que imponen situaciones experimentadas. Es decir, la capacidad percibida, a diferencia de la percepción de control, está relacionada con las intenciones y emociones excluidas en la TRA y la TPB, e incluidas en el TSE (Turan, 2012). La TPB explica el comportamiento previsto de sistemas que crean posturas específicas orientadas a sus evaluaciones, los principios y las expectativas para después terminar sus decisiones y por tanto su acción planeada, pero excluye la influencia de las variables de sistemas socioeconómicos-tecnológicos en las creencias. Por consiguiente, la Teoría de la Aceptación de la Tecnología (TAM por su acrónimo en inglés) es un modelo basado en los presupuestos de la TRA y la TPB. La TAM, cuyas variables disposicionales derivan de las variables cognitivas de la TPB y la TRA, incorporan variables exógenas como el clima organizacional que expliquen la aceptación o rechazo de una TIC más rápidamente.

En la TAM, la percepción de control, utilidad y facilidad de uso de la tecnología son definidas como el grado en que los individuos descubren que el uso de una tecnología no solo reduce su esfuerzo físico y mental, sino que mejora su desempeño. Ambas concepciones son intermediarias de la relación indirecta entre el uso de la tecnología. Incluso, la percepción de utilidad y facilidad de uso inciden en el empleo diferencial de Internet según el sexo y la edad del usuario (Mubarak \& Nycyk, 2017). La TAM se ha utilizado ampliamente para explicar la facilidad y los beneficios del uso de Internet (Park, 2009). En este proceso, las actitudes y las intenciones median el efecto perceptivo sobre la conducta. La TAM es más específico que la TRA o la TPB, pero también es más adecuado para explicar la relación individuo-tecnología, ya que abre la posibilidad de una explicación más detallada incluyendo factores externos a la cognición humana que se relacionan con el uso de internet.

\section{Estudios del uso de Internet}

En este apartado se incluyen los ejes, trayectorias y relaciones entre las variables que explican el uso intensivo de Internet. A partir del marco teórico y conceptual se establece la evidencia empírica. Las variables socioeconómicas o educativas se consideran exógenas a la cognición humana y afectan indirectamente al uso de las TIC. Es decir, la población más densa de tendencia, la inversión en la investigación y el coeficiente de inventiva se consideran variables que, en la interacción con el sexo, la edad, el nivel educativo y la situación económica podría poner fin al uso de Internet (Villa Zapata et al., 2015). En este sentido, los estudios psicológicos de la tecnología de afinidad de interacción especificaron relaciones indirectas entre variables externas y el uso de las TIC a través de cogniciones como percepciones, actitudes e intenciones. 
Son cuatro los niveles de interacción en los que habilidades y conocimientos están relacionados con las tecnologías de la información (Kiss de Alejandro, 2006). En el primer nivel, la interacción es en el hogar; en el segundo, la de los humanos con las tecnologías de la información; en el tercero, los componentes de TIC no son fundamento para el trabajo, el objetivo es solamente para reducir los costos; en el cuarto hay un enfoque dinámico en el que las habilidades y conocimientos son esenciales para implementar un nuevo sistema de producción, distribución y consumo. Este último nivel está relacionado con la aceptación, implementación, capacitación y producción de ventajas competitivas y estándares de certificación.

Los estudios psicológicos de crecimiento de las TIC consideran como exógeno económico y tecnológico al que determina las variables endógenas cognitivas y conductuales. Es decir, para acceder a las TIC, las organizaciones implementan programas de formación y capacitación entre los empleados para potenciar su desempeño a través de sus percepciones, actitudes e intenciones. Por lo tanto, la literatura muestra la prevalencia de ocho factores: 1) selectividad (Cassany \& Hernández, 2012), 2) compatibilidad (García Alonso, 2007), 3) computabilidad (Morales-Luna, 2011), 4) accesibilidad (Peraza et al., 2017), 5) extensión (Pifarré, 2013), 6) acumulación (Colle, 2013), 7) ansiedad (Cañón Buitrago et al., 2016) y 8) adicción (Sánchez-Carbonell et al., 2008), que han sido confirmados en estudios, actualizado y especificado en la materia, aunque esta última parece evidenciar más una externalización que una internalización de patologías (King, 2016). La revisión y discusión de este estado del arte permitió modelar sus relaciones como una contribución central a la disciplina (ver Tabla 1).

Tabla 1. Estado del conocimiento

Table 1. State of the art

\begin{tabular}{|c|c|c|c|c|}
\hline Año & Autor & Concepto & Indicadores & Modelado \\
\hline 2006 & Juarros & Selectividad & $\begin{array}{c}\text { Restricción y apertur } \\
\text { a }\end{array}$ & Selectividad $\leftarrow$ Uso de Internet \\
\hline 2009 & Serrano & Accesibilidad & $\begin{array}{l}\text { Facilidad, equidad y } \\
\text { representatividad }\end{array}$ & $\begin{array}{c}\text { Usabilidad } \rightarrow \text { Uso de } \\
\text { Internet } \leftarrow \text { Accesibilidad }\end{array}$ \\
\hline 2011 & $\begin{array}{l}\text { Yuslihasri } \\
\text { et al. }\end{array}$ & Compatibilidad & Aceptación y rechazo & Utilidad $\rightarrow$ Innovación $\leftarrow$ Compatibilidad \\
\hline 2011 & $\begin{array}{l}\text { Akin y } \\
\text { Iskender }\end{array}$ & Ansiedad & Unidimensional & Ansiedad $\rightarrow$ Adicción $\leftarrow$ Estrés \\
\hline 2012 & $\begin{array}{c}\text { De la } \\
\text { Cueva } \\
\text { González- } \\
\text { Cotera }\end{array}$ & Extensionalidad & Libertad y expresión & Extensionalidad $\leftarrow$ Uso de Internet \\
\hline 2015 & $\begin{array}{c}\text { Carreón et } \\
\text { al. }\end{array}$ & Computabilidad & Utilidad y facilidad & Uso de Internet $\rightarrow$ Computabilidad \\
\hline 2016 & $\begin{array}{l}\text { Rey- } \\
\text { Moreno y } \\
\text { Medina } \\
\text { Molina }\end{array}$ & Adiccion & $\begin{array}{l}\text { Riesgo negativo y } \\
\text { positivo }\end{array}$ & Uso de Internet $\rightarrow$ Asicción \\
\hline 2018 & $\begin{array}{l}\text { Gupta et } \\
\text { al. }\end{array}$ & $\begin{array}{c}\text { Acumulativament } \\
\mathrm{e}\end{array}$ & $\begin{array}{c}\text { Almacenamiento y } \\
\text { transmisión }\end{array}$ & Acumulativatividad $\rightarrow$ Uso de Internet \\
\hline
\end{tabular}


Es posible observar relaciones reflexivas más que determinantes del uso de Internet con respecto a las ocho dimensiones mostradas en la Tabla 1. Esto es así porque se asume evidencia empírica insuficiente entre las trayectorias de las dimensiones, aunque se detallan correlaciones entre ellas con un factor común: el uso de Internet.

El modelado de extensionalidad predice el uso del dispositivo como variable externa al sistema de adopción de la tecnología. La extensionalidad se asoció con otras siete variables para poder observar la emergencia de un factor común que la literatura identifica como uso intensivo (Cabero Almenara et al., 2016, p. 22). Las futuras líneas de investigación sobre los avances tecnológicos en la búsqueda de datos, así como su procesamiento, anticiparán escenarios de uso intensivo de tecnologías, dispositivos y redes de información electrónica.

La relación es formativa en el caso del uso de la tecnología basada en la accesibilidad (facilidad de uso). Si bien esta relación fue directa, también fue negativa y significativa (Juwita Siregar et al., 2017). El uso de la tecnología se refleja en la facilidad de acceder a ella, considerada como un aprendizaje continuo y sistemático de pruebas de aciertos y errores. En culturas latinas como México, esta práctica es hegemónica en comparación con la formación y educación de culturas postindustriales. Las líneas de investigación que aluden a las diferencias significativas entre culturas basadas en la sistematización del aprendizaje tecnológico versus culturas enfocadas en la transferencia permitirán anticipar la formación y el desempeño laboral a través de la tecnología.

La accesibilidad a la tecnología afectó negativamente su uso y se correlacionó con los factores de extensionalidad (Koul \& Eydahi, 2018). En consecuencia, una línea de investigación relacionada con la observación de la experiencia con la tecnología establecerá un modelo de acuerdo con la formación del capital humano y los usos tecnológicos para intensificar la búsqueda de información actualizada y especializada según lo exigido por la crisis de salud causada por el SARS-CoV-2 y la enfermedad de COVID-19.

La accesibilidad (facilidad, equidad y representatividad) y extensionalidad (libertad, expresión) son factores que la literatura ha modelado como formativos del uso intensivo de la tecnología. Dado que ambos factores son dimensiones reflejantes, las líneas de investigación en torno a la confirmación del modelo predecirán la relación entre el aula tradicional y el aula laboral. El estudio del uso intensivo de la tecnología es de vital importancia para explicar el fracaso o éxito del capital académico y profesional en el ámbito laboral.

\section{Modelamiento de las dimensiones del uso de Internet}

Siguiendo la teoría de modelos de ecuaciones estructurales en su formación o reflexión, y asumiendo que el uso de internet está conformado de varios indicadores que la literatura identifica como selectividad, utilidad, accesibilidad, usabilidad, compatibilidad, extensionalidad, computabilidad, acumulatibilidad, ansiedad y adicción, se propone el modelamiento del uso de Internet a partir de estas ocho variables manifiestas porque configuran variables manifiestas (Simonetto, 2012).

El fundamento teórico para el uso de Internet refleja de manera intensiva las ocho dimensiones sustraídas de la literatura publicada entre 2006 y 2020. Es un fenómeno latente, donde su modo reflexivo puede excluir algún factor y no alterar su estructura de relaciones; es decir, no cambiaría su 
revelación de latente a observable. En otras palabras, las consecuencias del uso de Internet, cuando se reflejan en cada una de las ocho dimensiones planteadas en el estado del conocimiento, pueden interpretarse como partes concomitantes de un fenómeno latente. En cambio, si estos factores se establecieran como determinantes del uso de Internet, se producirían modificaciones en la interpretación del modelo, lo que podría plantearse como un fenómeno que ha sido determinado por ocho factores en el que se espera que dichas variables aumenten. Este no es el caso del modelo reflexivo, porque cuanto más se consolide una estructura de factores que refleje el uso de Internet, más válido tendrá el instrumento que mida estas relaciones entre variables.

\section{METODOLOGÍA}

\section{Diseño}

Un estudio de corte correlacional, transversal y psicométrico.

\section{Muestra}

Selección intencional de 253 estudiantes de una universidad pública de México. El criterio de inclusión fue haber sido inscrito en el laboratorio de computación, pertenecer a una red social y buscar información para la elaboración de tareas, trabajos, pasantías, exposiciones, dinámicas, tesis o informes de investigación. La muestra estuvo conformada por 130 mujeres con un promedio de edad de 19.5 años ( $s=3.15$ años) y 123 hombres con un promedio de edad de 22.5 años ( $s=4.26$ años).

\section{Instrumento}

Consta de ocho dimensiones reportadas en la literatura: selectividad («Uso Internet para conocer la COVID-19»), compatibilidad («Uso Internet para prevenir la COVID-19»), computabilidad («Uso Internet por los registros de casos COVID-19»), accesibilidad («Uso Internet para revisar las experiencias con la COVID-19»), extensionalidad («Uso Internet para anticipar los rebrotes COVID19»), acumulatividad («Uso Internet porque almaceno datos sobre la COVID-19», ansiedad («Uso Internet por la actualización de la COVID-19») y adicción («Uso Internet por miedo a la COVID-19»). Cada dimensión fue medida a través de 4 ítems, los cuales contaron con 5 opciones de respuesta que iban desde el valor de 0: nada probable hasta el valor de 5: bastante probable.

\section{Proceso}

Se realizó un análisis Delphi para la validez del contenido o codificación de los ítems a partir de las definiciones operacionales de los mismos. El análisis consistió de 3 fases. En la primera, jueces expertos en la temática de uso de Internet calificaron las propuestas de los ítems; en la segunda fase, se compararon las calificaciones dadas por los expertos, en la tercera y última fase, se establecieron acuerdos y reconsideraciones. Una vez se completó el análisis Delphi, se procedió a aplicar la encuesta a las personas que cumplieron con los criterios de inclusión mencionados anteriormente. A cada participante del estudio se le pidió su consentimiento informado y se le indicó la finalidad del 
estudio. Los datos fueron analizados con ayuda del paquete de Estadística para Ciencias Sociales (SPSS, por sus siglas en inglés versión 20.0).

\section{Análisis}

Se estimaron los parámetros de distribución, fiabilidad y validez a fin de poder observar la consistencia interna del instrumento y la convergencia de las respuestas en factores. Se observó la estructura de trayectorias y relaciones mediante matrices de correlaciones y covarianzas, así como mediante un modelo de ecuaciones estructurales.

\section{Interpretación}

Para medir la consistencia interna se calculó el Alfa de Cronbach. Valores mayores de 0.6 dan cuenta de un buen indicador en este aspecto. En el caso de la validez de constructo se utilizó la correlación entre el ítem y el factor. Valores entre 0.3 y 0.9 son generalmente aceptados, ya que valores inferiores suponen la incidencia de otros factores en el ítem y los valores superiores al umbral se identifican como colineales. En el caso de correlaciones, los valores cercanos a cero se asumieron como evidencia de relación nula y los próximos a la unidad tanto negativa como positiva fueron contabilizados como evidencia de colinealidad. Respecto a las covarianzas, valores cercanos a cero fueron asumidos como evidencia de un factor común y los aproximados a la unidad como influencia unilateral de un factor común. En cuanto a los parámetros de ajuste, el nivel de significancia de ji al cuadrado permite comparar la estructura factorial del modelo teórico con la estructura factorial de los datos. Para los índices de bondad de ajuste como el GFI y el SRMR, valores por encima de 0.9 y por debajo de 0.08, respectivamente, son adecuados; al igual que $\chi 2$ / g.l < 3 (Hu \& Bentler, 1999; McClintock, Lau y Miller, 2016).

\section{RESULTADOS}

La Tabla 2 muestra los valores de parámetros que midieron las relaciones entre los indicadores con respecto a los factores. Se aprecia que están en el umbral esperado, corroborando la hipótesis de fiabilidad, la cual indica que convergerán los ítems en una subescala si estos miden rasgos de un concepto que los encuestados asimilan como tal y no lo confunden con otro rasgo de otro concepto. Por consiguiente, el instrumento puede ser empleado en otros contextos, escenarios y muestras midiendo los rasgos de un modo consistente. En el caso de la medición del uso de Internet, estos valores sugieren que la muestra encuestada distingue, cuando menos, ocho factores que explicarían los motivos y la necesidad de obtener información relativa a la pandemia. 
Tabla 2. Descriptivos del instrumento

Table 2. Instrument descriptions

\begin{tabular}{|c|c|c|c|c|c|c|c|c|c|c|c|}
\hline 1 & $M$ & SD & $A$ & $\mathrm{~F} 1$ & $\mathrm{~F} 2$ & F3 & $\mathrm{F} 4$ & F5 & F6 & F7 & F8 \\
\hline I1 & 1.03 & 0.49 & 0.782 & 0.381 & & & & & & & \\
\hline 12 & 1.06 & 0.39 & 0.761 & 0.382 & & & & & & & \\
\hline 13 & 1.07 & 0.29 & 0.703 & 0.392 & & & & & & & \\
\hline 14 & 1.19 & 0.31 & 0.741 & 0.391 & & & & & & & \\
\hline 15 & 3.10 & 0.52 & 0.742 & & 0.390 & & & & & & \\
\hline 16 & 3.04 & 0.39 & 0.732 & & 0.491 & & & & & & \\
\hline 17 & 3.17 & 0.69 & 0.790 & & 0.330 & & & & & & \\
\hline 18 & 3.47 & 0.25 & 0.762 & & 0.410 & & & & & & \\
\hline 19 & 1.06 & 0.51 & 0.721 & & & 0.482 & & & & & \\
\hline 110 & 1.01 & 0.83 & 0.752 & & & 0.482 & & & & & \\
\hline 111 & 1.83 & 0.93 & 0.743 & & & 0.410 & & & & & \\
\hline 112 & 1.27 & 0.62 & 0.761 & & & 0.441 & & & & & \\
\hline 113 & 1.20 & 0.63 & 0.703 & & & & 0.518 & & & & \\
\hline 114 & 1.17 & 0.84 & 0.762 & & & & 0.529 & & & & \\
\hline 115 & 1.28 & 0.85 & 0.793 & & & & 0.502 & & & & \\
\hline 116 & 1.62 & 0.19 & 0.751 & & & & 0.481 & & & & \\
\hline 117 & 1.69 & 0.29 & 0.703 & & & & & 0.518 & & & \\
\hline 118 & 1.30 & 0.38 & 0.751 & & & & & 0.382 & & & \\
\hline 119 & 1.64 & 0.41 & 0.703 & & & & & 0.493 & & & \\
\hline 120 & 1.02 & 0.62 & 0.762 & & & & & 0.513 & & & \\
\hline 121 & 1.29 & 0.47 & 0.703 & & & & & & 0.391 & & \\
\hline 122 & 1.63 & 0.35 & 0.761 & & & & & & 0.495 & & \\
\hline 123 & 1.03 & 0.25 & 0.703 & & & & & & 0.405 & & \\
\hline 124 & 1.83 & 0.38 & 0.704 & & & & & & 0.506 & & \\
\hline 125 & 0.38 & 0.05 & 0.761 & & & & & & & 0.381 & \\
\hline 126 & 0.49 & 0.07 & 0.703 & & & & & & & 0.406 & \\
\hline 127 & 0.16 & 0.08 & 0.703 & & & & & & & 0.591 & \\
\hline 128 & 0.39 & 0.04 & 0.762 & & & & & & & 0.471 & \\
\hline 129 & 0.82 & 0.04 & 0.775 & & & & & & & & 0.595 \\
\hline 130 & 0.95 & 0.05 & 0.783 & & & & & & & & 0.405 \\
\hline 131 & 0.71 & 0.08 & 0.792 & & & & & & & & 0.591 \\
\hline 132 & 0.39 & 0.05 & 0.761 & & & & & & & & 0.606 \\
\hline
\end{tabular}

Fuente: elaboración propia con los datos del estudio; I = ítem, M = Media o Promedio, DE = Desviación Estándar, A = Alfa de Cronbach quitando el valor del item. Curtosis general =2,47; Bootstrap $=0.000 ; \mathrm{KMO}=0.601 ; \mathrm{X} 2=12.35(24 \mathrm{~g}) \mathrm{p}=$ 0.000; F1 = Selectividad (18\% de varianza explicada); F2 = Compatibilidad (13\% de la varianza explicada); F3 = Computabilidad ( $10 \%$ de varianza explicada); F4 = Accesibilidad ( $7 \%$ para varianza explicada); F5 = Extensionalidad ( $5 \%$ de la varianza explicada); F6 = Acumulatividad ( $3 \%$ de la varianza explicada); F7 = Ansiedad ( $2 \%$ de la varianza explicada) y F8 = Adicción ( $1 \%$ de la varianza explicada). Todos los ítems incluyen cinco opciones de respuesta: 0 = nada probable, $1=$ muy improbable, 2 = poco improbable, 3 = poco probable, 4 = muy probable, 5 = bastante probable. 
Establecidos los ocho factores que explicaron el $59 \%$ de la varianza total, sus relaciones sugieren una estructura de trayectorias que reflejarían un factor común identificado como uso de Internet (ver Tabla 3). Se trata de ramificaciones explicativas de búsqueda, procesamiento y difusión de información relativa a la pandemia del SARS-CoV-2 y la enfermedad COVID-19 difundidas en los medios por el Estado. En tal proceso, la asimilación de conferencias, boletines y entrevistas con expertos se gesta en ocho dimensiones preponderantes. De este modo, la selectividad de datos explica el mayor porcentaje del uso de Internet ante la crisis sanitaria y la adicción explica un mínimo porcentaje de esa agenda epidémica. En este sentido, la muestra encuestada, considerada como audiencia de la propaganda estatal, procesa la información fragmentando sus contenidos y asociándolos con sus expectativas o necesidades.

Tabla 3. Estructura de relaciones entre factores

Table 3. Structure of relationships between factors

\begin{tabular}{|c|c|c|c|c|c|c|c|c|c|c|c|c|c|c|c|c|}
\hline & F1 & $\mathrm{F} 2$ & F3 & F4 & F5 & F6 & F7 & F8 & F1 & $\mathrm{F} 2$ & F3 & $\mathrm{F} 4$ & F5 & F6 & F7 & F8 \\
\hline F1 & 1.0 & $\begin{array}{c}.54 \\
*\end{array}$ & $\begin{array}{l}.62 \\
* *\end{array}$ & $\begin{array}{l}.40 \\
* *\end{array}$ & $\begin{array}{l}.50 \\
*\end{array}$ & $\begin{array}{l}.36 \\
* * *\end{array}$ & $.51 *$ & .52 & 1.9 & .67 & .53 & .48 & .63 & .65 & .68 & .64 \\
\hline F2 & & 1.0 & $\begin{array}{l}.48 \\
*\end{array}$ & $\begin{array}{l}.38 \\
* * *\end{array}$ & $\begin{array}{l}.63 \\
*\end{array}$ & $.49 *$ & $.66^{* *}$ & .60 & & 1.7 & .52 & .52 & .56 & .60 & .64 & .62 \\
\hline F3 & & & 1.0 & $.52 *$ & $\begin{array}{l}.59 \\
* *\end{array}$ & $\begin{array}{l}.50 \\
* *\end{array}$ & $.50 * * *$ & .63 & & & 1.6 & .63 & .49 & .53 & .58 & .58 \\
\hline F4 & & & & 1.0 & $\begin{array}{l}.42 \\
*\end{array}$ & $.47 *$ & $.48^{*}$ & .58 & & & & 1.8 & .44 & .59 & .55 & .55 \\
\hline F5 & & & & & 1.0 & $\begin{array}{l}.36 \\
* * \\
\end{array}$ & $.37 *$ & .57 & & & & & 1.5 & .42 & .63 & .53 \\
\hline F6 & & & & & & 1.0 & $.52 * *$ & .63 & & & & & & 1.3 & .67 & .47 \\
\hline F7 & & & & & & & 1.0 & .49 & & & & & & & 1.9 & .41 \\
\hline F8 & & & & & & & & 1.0 & & & & & & & & 1.6 \\
\hline
\end{tabular}

Fuente: elaboración propia con estudio de datos; F1 = Selectividad; F2 = Compatibilidad; F3 = Computabilidad; F4 = Accessibilidad; F5 = Extensionalidad; F6 = Acumulatividad; F7 = Ansiedad; F8 = Adicción: ${ }^{*} p<, 01 ;{ }^{* *} p<, 001 ;{ }^{* * *} p<$, 0001.

A fin de poder apreciar la estructura de trayectorias de relaciones entre los factores e indicadores, se procedió a realizar un análisis factorial confirmatorio. Es decir, relaciones entre factores e indicadores para advertir un factor común (véase Figura 1). Las respuestas de los encuestados develan que la información puede ser fragmentada en ocho dimensiones. Cada factor explica datos que por separado dan un sentido integral y al estar asociados develan un significado propagandístico. Se trata de una estructura factorial explicativa de la convergencia de opiniones en torno a la comunicación de riesgos ante la pandemia. Al no predominar un factor sobre otro se mantiene un equilibrio de asimilación de contenidos. 


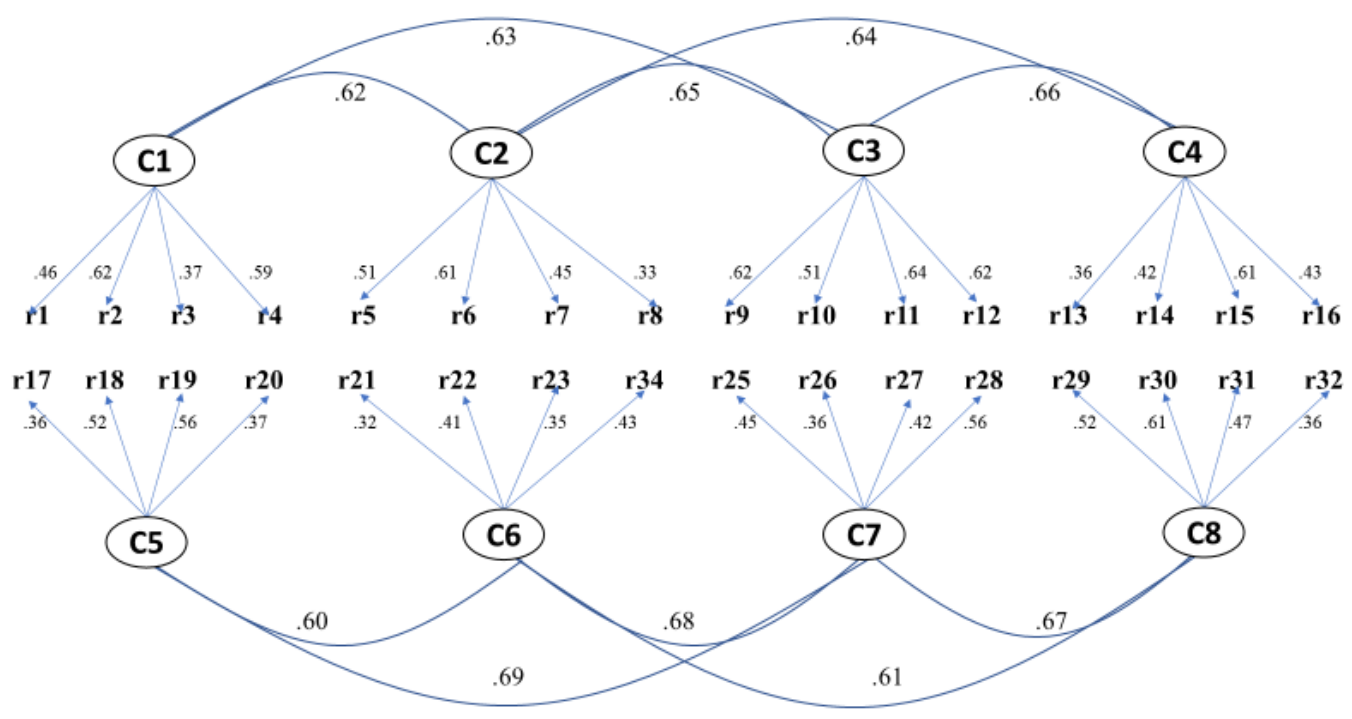

Figura 1. Análisis factorial confirmatorio

Figure 1. Confirmatory factor analysis

Fuente: elaboración propia con datos del estudio; F1 = Selectividad; F2 = Compatibilidad; F3 = Computabilidad; F4 = Accesibilidad; F5 = Extensionalidad; F6 = Acumulatividad; F7 = Ansiedad; F8 = Adicción.

La obtención de un $\chi 2 /$ g.l $=1.52<3$ sugiere el no rechazo de la hipótesis nula acerca de las diferencias entre los factores reportados en la literatura con respecto a las dimensiones observadas.

\section{DISCUSIÓN}

\section{Sobre panorama mundial del uso de Internet}

Debido a que la literatura advierte que en algunos países hay un crecimiento en la brecha digital entre personas con edades entre los 25 y los 34 años con respecto a otras edades (Leal Güemez \& Porras Duarte, 2019), el presente trabajo demostró que dicha brecha se extiende a lo largo de ocho factores, de los cuales la selectividad explica el mayor porcentaje de varianza expuesta (18\%). Lo anterior se da porque en ese rango de edad las habilidades de búsqueda de información son indispensables para el desempeño académico, profesional y laboral. Líneas de estudio concernientes a la autoeficacia computacional y las habilidades de búsqueda de información permitirán anticipar escenarios de formación del capital intelectual orientados hacia el desarrollo de sus criterios de selección informativa.

\section{Sobre el panorama nacional del uso de Internet}

En México, las ciudades con más de un millón de habitantes crecen simultáneamente con la demanda del servicio de Internet, considerando variables socioeconómicas, sociodemográficas y socioeducativas. El presente estudio ha observado que el uso de Internet como servicio demandado por jóvenes se estructura en torno a las habilidades computacionales para buscar y procesar información, siendo recomendable indagar sobre la relación entre estas variables con el desempeño 
académico, profesional o laboral a fin de poder advertir escenarios de desarrollo local y regional con base en el uso de tecnologías, dispositivos y redes informativas.

\section{Sobre la teoría del uso de Internet}

La literatura consultada ha establecido ejes, trayectorias y relaciones entre variables de orden valorativo, disposicional, motivacional, utilitario y de riesgo con respecto a habilidades, intenciones y uso de Internet. En el presente escrito se advierten ocho factores relativos a la percepción de control propuesta por la TPB, la capacidad percibida planteada por la TSE, la intención aludida en la TRA y la percepción de utilidad y facilidad de uso distintivas de la TAM. En estos ocho factores prevalece una estructura de relaciones entre ellas y sus indicadores que suponen un proceso deliberado, planificado y sistemático de adopción de la tecnología con base en las percepciones de control, utilidad, facilidad e intención de uso.

\section{Sobre los estudios del uso de Internet}

La accesibilidad (facilidad, equidad y representatividad) y extensionalidad (libertad, expresión) son factores reportados en la literatura como dimensiones que reflejan el uso de Internet que aluden a la búsqueda, procesamiento y difusión de información en situaciones límite, contingentes y riesgosas que se diseminan ante el uso de pares (Amadu et al., 2018). En la presente investigación ambos factores explicaron porcentajes menores al $10 \%$ de la varianza total, sugiriendo que se trata de subestructuras asociadas a otros procesos de recepción informativa como la necesidad de cognición o la probabilidad de elaboración cognitiva. Es decir, el uso de Internet como una moda informativa y difusora ante la pandemia es explicada por la necesidad informativa y la probabilidad de llevar a cabo el proceso de recepción y difusión si la tecnología, los dispositivos y las redes las reconocen como parte de una comunidad.

\section{Sobre el modelamiento de las dimensiones del uso de Internet}

En la propuesta de modelo para su contrastación empírica donde destacan las trayectorias reflejantes del uso de Internet, el presente trabajo ha encontrado una estructura factorial que sugiere el no rechazo de la hipótesis nula acerca de las diferencias significativas entre las relaciones teóricas y las empíricas. Es decir, la prueba empírica del modelo propuesto en la muestra encuestada sugiere la extensión del estudio. Líneas de investigación concernientes a la relación entre el uso de Internet y los ocho factores permitirán: 1) observar el efecto de la información relativa a la COVID-19 en la búsqueda, procesamiento y difusión por parte de internautas, 2) predecir el establecimiento de la agenda internauta, sus ejes, temas y rasgos distintivos de la pandemia, 3) sistematizar una revisión de la literatura alusiva al uso de Internet en situaciones excepcionales, 4) evaluar políticas sanitarias orientadas desde comunicación de riesgos.

\section{CONCLUSIONES}

La contribución de este estudio al campo de Ciencias Administrativas se da en términos del establecimiento de un instrumento confiable y válido para medir el uso de internet en época de 
COVID-19. En cuanto a los límites de diseño del estudio se recomienda extender la investigación de los componentes principales en el análisis factorial confirmatorio.

El objetivo del estudio fue diseñar y validar un instrumento para medir las dimensiones del uso de Internet en la era Covid-19, demostrando la prevalencia de ocho dimensiones que explicaron el 59\% de la varianza total, sugiriendo la contrastación del modelo en otros escenarios y muestras de estudios. La prueba empírica del instrumento permitirá evaluar políticas de mitigación y estrategias centradas en el confinamiento y distanciamiento social con orientación al aula virtual. La formación del capital intelectual en plataformas de enseñanza aprendizaje a distancia abren la discusión de la calidad de los procesos y productos académicos en la era Covid-19.

\section{CONFLICTOS DE INTERÉS}

Los autores declaran que no presentan conflictos de interés financiero, profesional o personal que pueda influir de forma inapropiada en los resultados obtenidos o las interpretaciones propuestas.

\section{CONTRIBUCIÓN DE AUTORES}

Para el desarrollo de este proyecto todos los autores han realizado una contribución significativa especificada a continuación:

Cruz García-Lirios: investigación documental, revisión de la literatura, curación de datos y análisis estadístico.

José Marcos Bustos-Aguayo: gestión, financiamiento, asesoría, revisión y corrección final de proyecto.

\section{REFERENCIAS}

Akin, A.; Iskender, M. (2011). Internet addiction and depression, anxiety and stress. International Online Journal of Educational Sciences, v. 3, n. 1, 138-148. URL

Amadu, L.; Syed Muhammad, S.; Mohammed, A.; Owusu, G.; Lukman, S. (2018). Using technology acceptance model to measure the use of social media for collaborative learning in Ghana. Journal of Technology \& Science Education, v. 8, n. 4, 321-336 https://doi.org/10.3926/jotse.383

Arribas, A.; Islas, O. (2009). Niños y jóvenes mexicanos en Internet. Razón y Palabra, n. 67. URL

Cabero Almenara, J. C.; Barrosso Osuna, J.; Llorente Cejudo, M. C. (2016). Technology acceptance model \& realidad aumentada: estudio en desarrollo. Revista Lasallista, v. 13, n. 2, 18-26. https://doi.org/10.22507/rli.v13n2a2 
Cañón Buitrago, S. C.; Castaño Castrillón, J. J.; Hoyos Monroy, D. C.; Jaramillo Hernández, J. C.; Leal Ríos, D. R.; Rincón Viveros, R.; Sánchez Preciado, E. A.; Ureña Calderón, L. S. (2016). Uso de Internet y su relación con la salud de estudiantes universitarios en la Ciudad de Manizales, (Caldas, Colombia), 2015-2016. Archivos de Medicina, v. 16, n. 2, 312-325. https://doi.org/10.30554/archmed.16.2.1735.2016

Carreón Guillén, J.; Hernández Valdés, J.; García Lirios, C. (2015). Contrastación de un modelo de ciberconsumo. Enseñanza e Investigación en Psicología, v. 20, n. 2, 218-226. URL

Cassany, D.; Hernández, D. (2012). ¿Internet: 1; escuela: 0? CPU-e, Revista de Investigación Educativa, v. 14, 126-141. URL

Cayla, J. A. (2009). Epidemias mediáticas: una reflexión para la salud pública. Gaceta Sanitaria, v. 23, n. 5, 362-364.https://doi.org/10.1016/i.gaceta.2009.09.002

Cervantes-López, M. J.; Peña-Maldonado, A. A.; Ramos-Sánchez, A. (2020). Use of information and communication technologies as a support tool for medical students' learning. CienciaUAT, v. 15, n. 1, 162-171. https://doi.org/10.29059/cienciauat.v15i1.1380

Colesca, S. E.; Dobrica, L. (2008). Adoption and use of e-government services: The case of Romania. Journal of Applied Research \& Technology, v. 6, n. 3, 204-216. https://doi.org/10.22201/icat.16656423.2008.6.03.526

Colle, R. (2013). Prensa y Big data: el desafío de la acumulación y análisis de datos. Mediterránea, v. 4, n. 1, 1-8. https://doi.org/10.14198/MEDCOM2013.4.1.13

De la Cueva González-Cotera, J. (2012). Internet como entorno de la opinión pública: envolviendo los derechos fundamentales en derechos ordinarios. Revista Internacional de Pensamiento Político, v. 7, 93-115. $\underline{U R L}$

Drozdova, A. V. (2020). The Dichotomy of Public/Private in the New Media Space. Changing Societies \& Personalities, v. 4, n. 4, 441-456. http://doi.org/10.15826/csp.2020.4.4.110

Eddine Toudert, D. (2015). Brecha digital y perfiles de uso de las TIC en México: Un estudio exploratorio con microdatos. Culturales, v. 3, n. 1, 167-200. URL

García Alonso, J. C. (2007). Hacia una web accesible. Acimed, v. 15, n. 5, 1-11. URL

Gard, R. (2018). Open data privacy and security policy issues and its influence on embracing the Internet of things. First Monday, v. 23, n. 5. https://doi.org/10.5210/fm.v22i5.8166

Garduño Vera, R. (2004). La sociedad de la información en México frente al uso de Internet. Revista Digital Universitaria, v. 5, n. 8, 1-13. URL 
Gravili, G.; Benvenuto, M.; Avram, A.; Viola, C. (2018). The influence of the Digital Divide on Big Data generation within supply chain management. The International Journal of Logistics Management, v. 29, n. 2, 592-628. https://doi.org/10.1108/IJLM-06-2017-0175

Gupta, A.; Garg, D.; Kumar, P. (2018). Analysis of Students' Ratings of Teaching Quality to Understand the Role of Gender and Socio-economic Diversity in Higher Education. IEEE Transaction on Education, v. 61, n. 4. https://dx.doi.org/10.1109/TE.2018.2814599

Hu, L. T.; Bentler, P. M. (1999). Cutoff criteria for fit indexes in covariance structure analysis: Conventional criteria versus new alternatives. Structural Equation Modeling: A Multidisciplinary Journal, v. 6, n. 1, 1-55. http://doi.org/10.1080/10705519909540118

Instituto Nacional de Estadística, Geografía e Informática (2020). En México somos 126.014.024 habitantes: censo de población y vivienda 2020. $\underline{\text { UR }}$

Jiang, Y.; Chen, D.; Lai, F. (2010). Technological personal environmental (TPE) framework: A conceptual model for technology acceptance at the individual level. Journal of International Technology \& Information Management, v. 19, n. 3, 89-98. URL

Juarros, M. F. (2006). ¿Educación superior como derecho o como privilegio? Las políticas de admisión a la universidad en el contexto de los países de la región. Andamios, v. 3, n. 5, 69-90. https://doi.org/10.29092/uacm.v3i5.342

Juwita Siregar, J.; Aryanti Wardaya Puspokusumo, R. A.; Rahayu, A. (2017). Analysis of the affecting factors technology acceptance model in the application of knowledge management for small medium enterprises in industry creatives. Procedia Computer Science, v. 116, 500-508. https://doi.org/10.1016/j.procs.2017.10.075

King, D. (2016). Internet addictions. Cyberpsychology: Journal of Psychosocial Research on Cyberspace, v. 10, n. 3. https://doi.org/10.5817/CP2016-3-1

Kiss de Alejandro, D. M. (2006). Niveles de interacción en la comunicación de Internet. Ámbitos, n. 15, 45-57. URL

Kleine, D. (2009). The ideology behind the technology-Chilean microentrepreneurs and public ICT policies. Geoforum, v. 40, n. 2, 171-183. https://doi.org/10.1016/i.geoforum.2008.02.006

Koul, S.; Eydgahi, A. (2018). Utilizing technology acceptance model for driverless car technology adoption. Journal Technology Management Innovation, v. 13, n. 4, 37-46. https://doi.org/10.4067/S0718-27242018000400037

Lai, P. C. (2017). The literature review of technology adoption models and theories for the novelty technology. Journal of Information System \& Technology Management, v. 14, n. 1, 21-38. https://doi.org/10.4301/s1807-17752017000100002 
Leal Güemez, R.; Porras Duarte, S. T. (2019). Desarrollo digital en México. Denarious, v. 37, 13-47. https://doi.org/10.24275/uam/izt/dcsh/denarius/v2019n37/Leal

Liao, S.; Hong, J.-C.; Wen, M.-H.; Pan, Y.-C.; Wu, Y. (2018). Applying technology acceptance model (TAM) to explore user's behavioral intention to adopt a performance assessment system for ebook production. Journal of Mathematical Science \& Technology Education, v. 14, n. 10, em1601. https://doi.org/10.29333/ejmste/93575

Mayor-Ríos, J. A.; Pacheco-Ortiz, D. M.; Patiño-Vanegas, J. C.; Ramos-y-Yovera, S. E. (2019). Análisis de la integración del Big Data en los programas de contaduría pública en universidades acreditadas en Colombia. Revista CEA, v. 5, n. 9, 53-76. https://doi.org/10.22430/24223182.1256

McClintock, C. H.; Lau, E.; Miller, L. (2016). Phenotypic Dimensions of Spirituality: Implications for Mental Health in China, India, and the United States. Frontiers in Psychology, v. 7. http://doi.org/10.3389/fpsyg.2016.01600

Mecinas Montiel, J. M. (2016). The digital divide in Mexico: A mirror of proverty. Mexican Law Review, v. 9, n. 1, 93-102. https://doi.org/10.1016/j.mexlaw.2016.09.005

Morales-Luna, G. (2011). Computabilidad y computación cuántica: Revisión de modelos alternativos de computación. Ingeniería Industrial, v. 10, n. 2, 49-53. URL

Mubarak, F.; Nycyk, M. (2017). Teaching older people internet skills to minimize grey digital divides: Developed and developing countries in focus. Journal of Information, Communication and Ethics in Society, v. 15, n. 2, 165-178. https://doi.org/10.1108/JICES-06-2016-0022

Organización Mundial de la Salud (2021). Enfermedad por coronavirus (COVID-19). URL

Ozamiz, N.; Dosil, M.; Picaza, M.; Idoiaga, N. (2020). Niveles de estrés, ansiedad y depresión en la primera fase del brote del Covid-19 en una muestra recogida en el norte de España. Cuadernos de Saude Publica, v. 36, n. 4, 1-9. https://doi.org/10.1590/0102-311X00054020

Park, S. Y. (2009). An analysis of the technology acceptance model in understanding university students' behavioral intention to use e-learning. Educational Technology Society, v. 12, n. 3, 150162. URL

Peláez Herreros, O. (2009). Descripción y proyección de la esperanza de vida al nacimiento en México (1900-2050). Estudios Demográficos y Urbanos, v. 24, n. 2, 469-492. https://doi.org/10.24201/edu.v24i2.1341

Peraza, J.; Quiñonez, Y.; Lizárraga, C.; Olivarría, M.; Estrada, R.; Ortega, J. (2017). Haciendo el internet accesible, un paso más hacia la universalidad de la información. Computación, Informático, Biomédica y Electrónica, v. 6, n. 1, 61-75. URL 
Pifarré, M. J. (2013). Internet y redes sociales: un nuevo contexto para el delito. Internet, Derecho y Política, n. 16, 40-43. https://doi.org/10.7238/idp.v0i16.1985

Primabudi, A. M.; Samopa, F. (2017). Analysis of factor influencing purchase decision in online store. Case study: Game sales in online store. International Journal of Education \& Research, v. 5, n. 7, 277-288. URL

Rey-Moreno, M.; Medina Molina, C. (2016). Social capital in e services adoption. Psychology \& Marketing, v. 33, n. 12, 1151-1158 https://doi.org/10.1002/mar.20952

Sánchez-Carbonell, X.; Beranuy, M.; Castellana, M.; Chamarro, A.; Oberst, U. (2008). La adicción a Internet y el móvil: ¿̇moda o trastorno? Adicciones, v. 20, n. 2, 149-159.

https://doi.org/10.20882/adicciones.279

Serrano Mascaraque, E. (2009). Accesibilidad versus usabilidad web: evaluación y correlación. Investigación Bibliotecológica, v. 23, n. 48, 61-103.

https://doi.org/10.22201/iibi.0187358xp.2009.48.16970

Sevim, N.; Yüncü, D.; Eroğlu Hall, E. (2017). Analysis of the extend technology acceptance model in online travel products. Journal of Internet Applications and Management, v. 8, n. 2, 45-61. https://doi.org/10.5505/iuyd.2017.03522

Simonetto, A. (2012). Formative and reflective models: state of the art. Electronic Journal of Applied Statistical Analysis, v. 5, n. 3, 452-457. https://doi.org/10.1285/i20705948v5n3p452

Turan, A. H. (2012). Internet shopping behavior of Turkish customer: comparison of two competing models. Journal of Theoretical \& Applied Electronic Commerce Research, v. 7, n. 1, 77-93. https://doi.org/10.4067/S0718-18762012000100007

Villa Zapata, A. M.; Ramírez Salazar, K. P.; Tavera Mesías, J. F. (2015). Antecedentes de la intención de uso de los sitios WEB de compras colectivas. Revista EIA, v. 12, n. 24, 55-70. https://doi.org/10.14508/reia.2015.12.24.55-70

Wamuyu, P. K. (2017). Bridging the digital divide among low income urban communities. Leveraging use of Community Technology Centers. Telematics and Informatics, v. 34, n. 8, 1709-1720. https://doi.org/10.1016/i.tele.2017.08.004

Yasa, N. N. K.; Ratnaningrum, L. P. R. A.; Sukaatmadja, P. G. (2014). The application of technology acceptance model on Internet banking users in the city of Denpasar. Journal management Dan, v. 16, n. 2, 93-102. https://doi.org/10.9744/jmk.16.2.93-102

Yuslihasri, E.; Aminul Islam, Md.; Ku Daud, K. A. (2011). Factors That Influence Customers Buying Intention On Shopping Online. International Journal of Marketing Studies, v. 3, n. 1, 128-143. https://doi.org/10.5539/ijms.v3n1p128 
Zahid, H.; Haji Din, B. (2019). Determinants of intention to adopt e-government services in Pakistan: An imperative for sustainable development. Resources, v. 8, n. 3, 1-25. https://doi.org/10.3390/resources8030128

Zhang, S.; Li, F.; Xiao, J. J. (2020). Internet penetration and consumption inequality in China. International Journal of Consumer Studies, v. 44, n. 5, 407-422. https://doi.org/10.1111/ijcs.12575 